\title{
Ferromagnetic resonance for the quantification of superparamagnetic iron oxide nanoparticles in biological materials
}

This article was published in the following Dove Press journal:

International Journal of Nanomedicine

24 March 2010

Number of times this article has been viewed

\author{
Lionel F Gamarra ${ }^{1,2}$ \\ Antonio J daCosta-Filho ${ }^{3}$ \\ Javier B Mamani' \\ Rita de Cassia Ruiz ${ }^{4}$ \\ Lorena F Pavon' \\ Tatiana T Sibov' \\ Ernanni D Vieira ${ }^{3}$ \\ André C Silva' \\ Walter M Pontuschka ${ }^{5}$ \\ Edson Amaro Jr ${ }^{1,2}$ \\ 'Instituto Israelita de Ensino e \\ Pesquisa Albert Einstein, IIEPAE, São \\ Paulo, Brazil; ${ }^{2}$ Instituto de Radiologia, \\ Faculdade de Medicina, Universidade \\ de São Paulo, São Paulo, Brazil; \\ ${ }^{3}$ Instituto de Física de São Carlos, \\ Universidade de São Paulo, São \\ Carlos, Brazil; ${ }^{4}$ Instituto Butantan, \\ São Paulo, Brazil; ${ }^{5}$ nstituto de Física, \\ Universidade de São Paulo, São Paulo, \\ Brazil
}

Correspondence: Lionel F Gamarra Instituto Israelita de Ensino e Pesquisa Albert Einstein (IIEPAE), Sociedade Beneficente Israelita Brasileira Hospital Albert Einstein (SBIBHAE). Av. Albert Einstein, 627/70I, Piso Chinuch ( $2^{\circ}$. Subsolo), Morumbi, São Paulo/SP, Brazil, CEP 0565I-90I

Tel +55 I | 3747 | 366

Fax +55 II 38134334

Email Igamarra@einstein.br

\begin{abstract}
The aim of the present work is the presentation of a quantification methodology for the control of the amount of superparamagnetic iron oxide nanoparticles (SPIONs) administered in biological materials by means of the ferromagnetic resonance technique (FMR) applied to studies both in vivo and in vitro. The in vivo study consisted in the analysis of the elimination and biodistribution kinetics of SPIONs after intravenous administration in Wistar rats. The results were corroborated by X-ray fluorescence. For the in vitro study, a quantitative analysis of the concentration of SPIONs bound to the specific AC133 monoclonal antibodies was carried out in order to detect the expression of the antigenic epitopes (CD133) in stem cells from human umbilical cord blood. In both studies FMR has proven to be an efficient technique for the SPIONs quantification per volume unit (in vivo) or per labeled cell (in vitro).
\end{abstract}

Keywords: quantification, FMR, ferrofluid, biodistribution, nanoparticles

\section{Introduction}

Superparamagnetic iron oxide nanoparticles (SPIONs) are known to consist of ferromagnetic single domains with great potential for biological and medical applications. ${ }^{1-3} \mathrm{~A}$ considerable number of preliminary studies have been performed to address issues such as toxicity, ${ }^{4-6}$ kinetics of elimination and biodistribution of the nanoparticles in the different tissues, ${ }^{7-9}$ biocompatibility, ${ }^{9}$ cellular labeling, ${ }^{10-12}$ qualitative visualization of the tissue or the labeled cell, ${ }^{5,13-15}$ viability and cellular proliferation, ${ }^{5,6,14}$ differentiation assays, ${ }^{16,17}$ kinetics of the SPIONs-trapping capability, ${ }^{18}$ analysis of the qualitative biodistribution, ${ }^{19}$ efficiency of cellular labeling, ${ }^{6,13,14,16}$ and qualitative analysis of the iron content,, $50-22$ among others.

In biological and medical applications, an important point involved with the utilization of the SPIONs is the iron quantitative analysis. For example, in in vivo studies, it is necessary to determine: i) the quantity of iron that was absorbed or bound to the different tissues after SPIONs administration; ii) the iron absorption or elimination rate; iii) whether the amount of iron administered per volume unit is toxic or not to the tissue or to the cell; iv) and what is the ideal dose to obtain the desired effect in the particular application, among others. In applications of magnetic resonance imaging (MRI), these parameters will allow the contrast effect in the signal intensity to be determined, resulting in a better contrast control due to the presence of SPIONs. In in vitro studies the quantitative analysis of the SPIONs has the following fundamental objectives: i) determination of the relation between the signal intensity and the number of the labeled cells, as well as the signal intensity and the 
administered SPIONs concentration (taking into account the study of the toxicity of the contrast agent of cellular label); ii) determination of the ideal dose and the ideal time after the SPIONs administration (result of the study of the kinetics and the tissue SPIONs trapping capability) to obtain a good contrast for MRI; iii) determining the quantity of cells that can be detected in vivo or in vitro by MRI; and iv) to verify how the MRI signal intensity is related with the cellular proliferation and migration.

The objective of this work is to present the quantification methodology of SPIONs for the studies in biological materials by means of ferromagnetic resonance (FMR) both in vivo and in vitro. For the in vivo case, FMR was used to monitor the kinetics of ferrofluid elimination and biodistribution after the SPIONs administration, with the results corroborated by X-ray fluorescence (XRF) measurements. In the in vitro study, hematopoietic stem cells from human umbilical cord blood were labeled with SPIONs coupled to a specific AC133 monoclonal antibody in order to monitor the expression of the antigenic CD133 label.

\section{Experimental procedure Methodology of quantification}

The methodology here described is intended to provide a quantitative analysis of iron oxide nanoparticles in biological materials by means of FMR technique. The analysis allows one to have a better control of the SPIONs dose administered in biological materials. Such methodology can be summarized as follows:

a) Perform an initial qualitative visualization of the studied tissue in order to observe the SPIONs location as well as the conservation of the cells morphology, thus validating the posterior quantification analysis.

b) In the FMR spectrum, assign the peaks that specifically correspond to SPIONs, since other iron species that do not come from the administered SPIONs can also contribute peaks to the FMR spectrum. This identification is to be performed carefully by measurements of FMR spectra at different temperatures, taking into account the blocking temperature of the nanoparticles. For FMR measurements, room temperature is recommended because at higher temperatures changes that lead to irreversible damages in the samples can happen.

c) Build the calibration curve for the ferrofluid using different known concentrations which are proportional to the areas under the resonance absorption curves (doubly integrated FMR experimental spectrum) of the SPIONs. This curve will allow an indirect interpolation during the quantification process. The parameters of the FMR data acquisition used to construct the curve as well as the volume of the sample for all the concentrations must be equal.

d) After confirming the presence of the SPIONs in the tissues (desired target), assigning the SPIONs resonance line, and taking into account the calibration curve, the SPIONs quantification is performed for the different samples as follows:

- Carry out FMR measurements of the studied samples, where the parameters used for data acquisition (not necessarily the same of the calibration curve) and the volume of the samples must be equal.

- Take a FMR spectrum of a sample with known SPIONs concentration. Then, interpolate the value of the area under the absorption signal in the calibration curve in order to obtain a "factor" to be multiplied by the areas obtained from measured FMR spectra of the samples under study and thus to find the correspondent concentrations (indirect calibration).

This paper is concerned with the application of this method in two cases of quantification (in vivo and in vitro), where we describe the details and indicate the necessary precautions.

\section{Case I: in vivo study}

The process of SPIONs quantification in vivo was conducted in the study of the kinetics of elimination in the blood and biodistribution in the liver of the biocompatible ferrofluid constituted by $\mathrm{Fe}_{3} \mathrm{O}_{4}$ nanoparticles recovered with dextran, and administered via intravenous in Wistar rats. The FMR results were verified by the use of XRF technique.

\section{Ferrofluid: Contrast agent}

The biocompatible ferrofluid used in the in vivo study is an aqueous colloid of $\mathrm{Fe}_{3} \mathrm{O}_{4}$ SPIONs (Endorem, Guerbet, France) coated with low-molecular-weight dextran $(7-9 \mathrm{kDa}){ }^{23}$ the iron oxide crystal is $4.8-5.6 \mathrm{~nm}$, and the hydrodynamic diameter is $80-150 \mathrm{~nm}$. Each milliliter of Endorem contains: $11.2 \mathrm{mg}$ of iron, $5.6-9.1 \mathrm{mg} / \mathrm{mL}$ of dextran, $0.25-0.53 \mathrm{mg} / \mathrm{mL}$ of citric acid, and $61.3 \mathrm{mg} / \mathrm{mL}$ of $\beta$-D-manitol $\left(\mathrm{C}_{6} \mathrm{H}_{14} \mathrm{O}_{6}\right)$.

\section{Protocol for SPIONs administration}

A number of 16 male Wistar rats (90 days old) with average body weight of $420 \mathrm{~g}$ was selected. After alimentary starving, the animals were weighed and anesthetized with ketamine chlorohydrate $(100 \mathrm{mg} / \mathrm{kg})$ and chlorohydrate of xilazine 
$(10 \mathrm{mg} / \mathrm{kg})$, mixed in the same syringe and injected intramuscularly. After 20 minutes, a solution of the biocompatible ferrofluid, at the concentration of $15 \mu \mathrm{mol}$ of Fe $/ \mathrm{kg}$ of body weight, was slowly injected (about 10 minutes).

Samples of blood were collected during the periods of $5,15,30,45,60,90$, and $180 \mathrm{~min}$, and the animals were subsequently sacrificed in a $\mathrm{CO}_{2}$ chamber. Samples of liver were collected after each sacrifice. The control was performed with rats that were not injected with the ferrofluid.

The collected samples were then lyophilized in an Edwards equipment, model E3M8-Modulyo, in order to achieve an optimum homogeneity for the subsequent analyses of the kinetics of elimination and biodistribution of the ferrofluid using the techniques of FMR and XRF.

\section{Quantification protocol}

\section{Visualization of the SPIONs in the studied tissue}

The samples of liver were fixed in Bouin and included in Paraplast $^{\circledR}$ (McCormick Scientific, St. Louis, MO, USA). For the histological analysis, incisions ( $5 \mu \mathrm{m}$ thick) were colored with hematoxylin and eosin (HE). For the detection of ferric ions the cuts were submitted to the reaction with Prussian blue (Perls method) and countercolored with a solution of safranine. The analysis and the documentation of the cuts were performed in a light microscope (Axiophot; Carl Zeiss, Oberkochen, Germany).

\section{Identification of the SPIONs in the samples by FMR}

In the study of SPIONs elimination and biodistribution, it was necessary to determine the iron entities present in the liver and blood of the rats after the administration of the ferrofluid. The FMR spectra were acquired using lyophilized samples of the liver and blood. In the search for the best FMR signal, the spectra were obtained at different temperatures using a spectrometer operating at X-band. The FMR measurements were performed using an ELEXSYS E580 spectrometer (Bruker BioSpin, São Paulo, Brazil) operating at $9.4 \mathrm{GHz}$. The samples were placed in a quartz tube which was in turn positioned in the center of a rectangular microwave cavity operating in the TE102 mode. To perform experiments at different temperatures $(4,100$, and $298 \mathrm{~K})$ with liver and blood samples, the FMR spectrometer was also equipped with a helium gas flow cryostat (continuous flow cryostat CF935; Oxford Instruments, Abingdon, UK) mounted inside the microwave cavity. The temperature of the sample was controlled with an ITC503 Oxford Instruments temperature control unit and the spectra were collected after the samples reached thermal equilibrium. In order to avoid saturation and distortion of the FMR signal, the following experimental parameters were used: center field 2,500 Gauss; sweep width 4,900 Gauss; modulation amplitude, 3.0 Gauss; modulation frequency, $100 \mathrm{kHz}$; microwave power $10 \mathrm{~mW}$; conversion time, $81.92 \mathrm{~ms}$; time constant, $20.48 \mathrm{~ms}$.

\section{Calibration curve}

The calibration curve shown in Figure 1 was constructed in order to determine the average number of SPIONs per volume unit of the samples being studied. Samples of the ferrofluid (Endorem) were prepared with colloidal suspensions at different concentrations varying from 0.3 to $101 \times$ $10^{13} \mathrm{SPION} / \mathrm{mm}^{3}$. A $2 \mu \mathrm{L}$ volume of each sample was inserted into capillary quartz tubes for the acquisition of the FMR spectra as shown in Figure 1A. Each point in the calibration curve was the result of three scans and the plotted value represents the average of these measurements.

Since the derivative of the absorption curve is measured in an FMR experiment, the intensity of each FMR spectrum is determined from the double integration of the experimental resonance peaks (in our case, the peak at $\mathrm{g}=2.1$ was assigned to the iron-containing SPIONs). The g-value was obtained from the relation $\mathrm{g}=\left(h \mathrm{v} /\left(\beta \mathrm{H}_{0}\right)\right)=(2.12 \pm 0.02)$, with $v=9.428 \mathrm{GHz}$, where $h$ is the Planck constant, $v$ is the microwave frequency and $\beta$ is the Bohr magneton. The intensity of the FMR spectrum at $g=2.1$ is proportional to the number of iron particles per $\mathrm{mm}^{3}$ (concentration of SPIONs). The values obtained from the FMR intensities associated with the iron concentrations were plotted and the calibration curve was obtained (solid line in Figure 1). The uncertainty in each experimental calibration point was estimated to be in the range $0.3-2.0 \times 10^{11}$, which thus cannot be seen in the same scale used in Figure 1.

In order to check the results obtained in vivo by FMR a study was conducted using the technique of XRF and calibration curves were also built for samples of the ferrofluid Endorem as well as the standard samples $1577 \mathrm{~b}$ and $1548 \mathrm{a}$ of the National Institute of Standards and Technology. The XRF powder calibration curve relates the characteristic line of iron (shown in Figure 1B) versus the concentration of the iron particles exhibited by the dashed line in Figure 1. The XRF measurements were performed using EDX-980 equipment (Shimadzu, São Paulo, Brazil).

The fraction of iron due to administered SPIONs was obtained from the differences between the XFR iron line in the control sample and the same line in the studied sample thus yielding a real value of the iron content in the organs being studied, and due only to the administered substance. The same procedure was adopted in the FMR studies. 


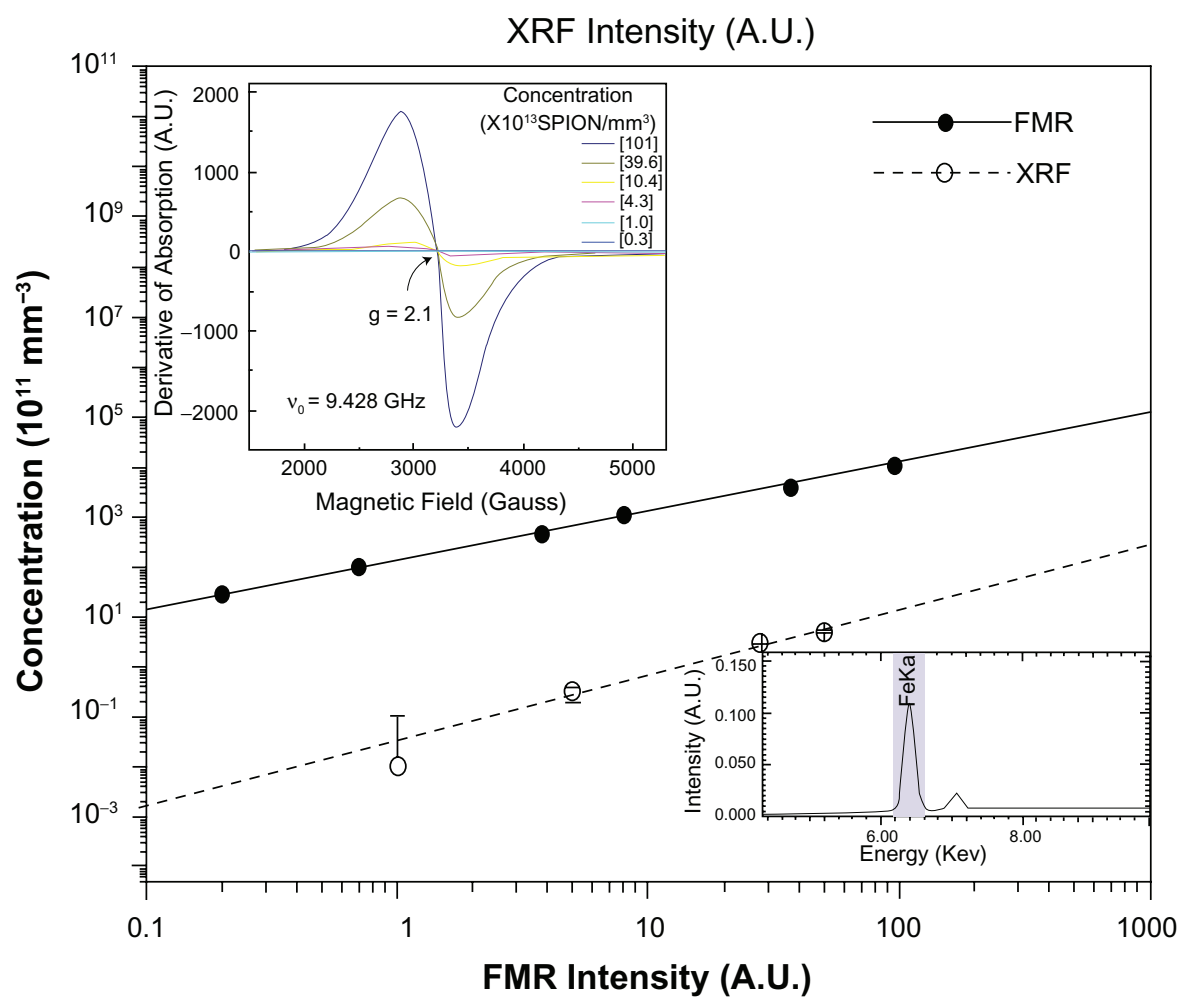

Figure I FMR (solid line) and XRF (dashed line) calibration curves of the SPIONs related to the area under the resonance line at $g=2.1$ and the characteristic FeKa line, respectively. In the inset $\mathbf{A}$ ) there are seen typical FMR spectra for different concentrations of iron, corresponding to the characteristic line of magnetite contained in the ferrofluid Endorem ${ }^{\mathrm{TM}}$. Inset $\mathbf{B}$ ) shows the characteristic FeKa line of XRF, proportional to the iron concentration in the sample.

Abbreviations: FMR, ferromagnetic resonance; SPIONs, superparamagnetic iron oxide nanoparticles; XRF, X-ray fluorescence.

\section{FMR measurements}

The FMR spectra were acquired using lyophilized samples of the liver and blood. For the purpose of performing the indirect interpolation was used a volume of $0.1 \mathrm{~mL}$ of the ferrofluid (Endorem) of known concentration $\left(5 \times 10^{13}\right.$ particles $\left./ \mathrm{mm}^{3}\right)$.

\section{Case II: in vitro study}

The in vitro quantification of SPIONs by means of FMR comprised the labeling of CD $133^{+}$stem cells from the blood of human umbilical cord. The SPIONs are coupled to AC133 monoclonal antibody, which recognizes the antigenic epitope CD133. The $\mathrm{CD}_{133^{+}}$cells from human cord blood could represent a primary step to gain access to a stem pool useful for therapeutic hematopoietic transplants, stem cell expansion for clinical purposes and pre-clinical research on tissue regenerating therapies.

\section{Ferrofluid cellular label}

CD133 cells were labeled by using the AC133 monoclonal antibody coupled to the SPIONs $-\mathrm{Fe}_{3} \mathrm{O}_{4}-$ (Miltenyi Biotec, Bergisch Gladbach, Germany) with average particle diameter of $9 \mathrm{~nm}$. These nanoparticles were dispersed in a colloidal suspension of a ferrofluid containig $0.1 \%$ of gelatine, $0.05 \%$ of sodium azide, and an iron content of $200 \mu \mathrm{g} / \mathrm{mL}$.

\section{Protocol for labeling CDI 33 cells with SPIONs}

Blood from umbilical cord cells were obtained from donor volunteers $(\mathrm{n}=5)$ after written consent (CEP-IEPAE No.105/02). Mononuclear cells were purified by densitygradient centrifugation using Ficoll-Paque ${ }^{\mathrm{TM}}$ Plus (GE Healthcare, Chalfont St. Giles, UK). ${ }^{24}$

After the separation of the mononuclear cells, the $\mathrm{CD}_{133^{+}}$cell population was purified using anti-CD133 $\mathrm{mAb}$-coupled magnetic beads (Miltenyi Biotec), according to the manufacturer's instructions. Briefly, to each fraction of $10^{8}$ cells was added $100 \mu \mathrm{L}$ of Fc-receptor. After that, the cells were labeled with $100 \mu \mathrm{L}$ of CD133 microbeads (final volume: $500 \mu \mathrm{L} / 10^{8}$ total cells). The cells were incubated for 30 minutes at $4-8^{\circ} \mathrm{C}$; after the incubation, the cells were washed and resuspended in $500 \mu \mathrm{L}$ of buffer for every $10^{8}$ cells.

The cells, magnetically labeled with antibodies antiCD133 microbeads, were separated on a column placed in a magnetic field. In this process, the magnetic-labeled $\mathrm{CD}_{133^{+}}$cells were retained in the column, whereas the 
unlabeled $\mathrm{CD} 133^{-}$cells passed through. After the removal of the column from the magnetic field, the magnetically retained $\mathrm{CD} 133^{+}$cells could be eluted as the positively selected fraction.

\section{Quantification protocol for the magnetic labeling}

The quantification methodology followed the same general steps presented above and are described below for the in vitro case.

\section{Visualization of the SPIONs in the CDI $33^{+}$cells}

After separation, the $\mathrm{CD} 133^{+}$cell population was fixed using $0.5 \%$ glutaraldehyde in $0.2 \mathrm{M}$ cacodylate buffer for two hours at $4^{\circ} \mathrm{C}$ following the routine treatment. The material was then transferred to pure Epon resin and incubated at $60^{\circ} \mathrm{C}$ for 72 hours, until it was completely polymerized. The ultrathin sections, obtained with the aid of an ultramicrotome, were placed on copper grids and stained with uranyl acetate and lead citrate. The grids were studied and photographed with a transmission electron microscope (PHILIPS CM100; Philips, Amsterdam, The Netherlands).

\section{Calibration curve}

The calibration curve shown in Figure 2 was built using several FMR absorption spectra measured at room temperature (inset of Figure 2) and containing samples with different concentrations with the main goal of determining the average number of SPIONs per cell. Samples with the following concentrations of the cellular label were prepared in colloidal suspension: $0.040 \pm 0.002 ; 0.68 \pm$ $0.04 ; 1.25 \pm 0.30 ; 1.90 \pm 0.04 ; 2.76 \pm 0.03$; and $3.58 \pm$ $0.02 \mathrm{mM}$. The uncertainties in the FMR spectral intensity (area under the $\mathrm{g}=2.1$ absorption curve) varied in the range of a few arbitrary units and hence could not be represented in the calibration curves. The volumes of all samples used to obtain the FMR spectra were $2 \mu \mathrm{L}$. Similarly, as done in the in vivo procedure, each measured point was the average value of three scans.

\section{FMR measurements of the labeled cells}

After labeling, the $\mathrm{CD} 133^{+}$cells were collected in a volume of $2 \mu \mathrm{L}$ for the acquisition of their FMR absorption spectra. The indirect interpolation was performed using a sample of

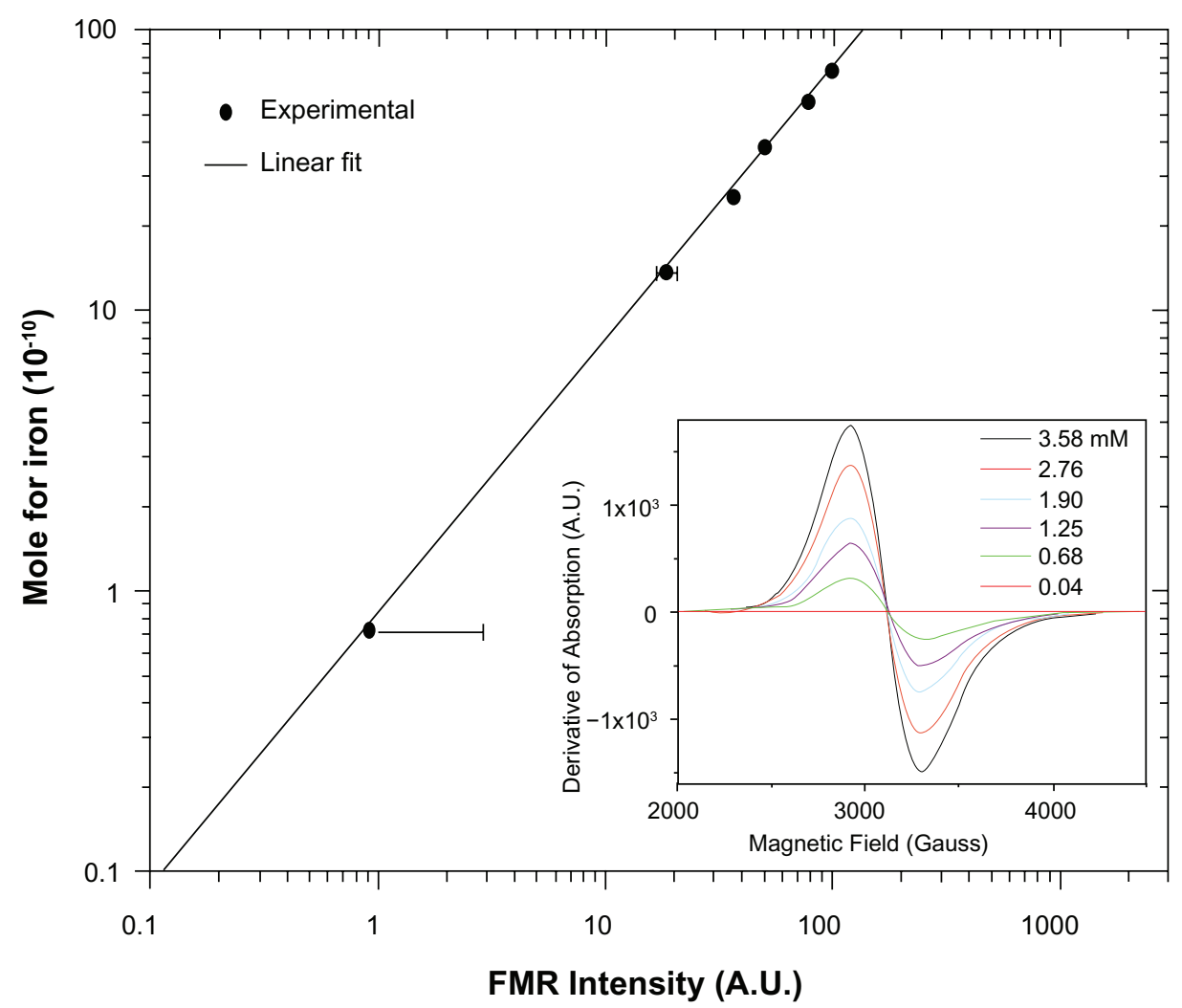

Figure 2 FMR calibration curve of the SPIONs concentration related with the area under the resonance absorption curve at $g=2.1$. In the inset there are presented the typical FMR spectra at different concentrations of magnetite contained in the ferrofluid.

Abbreviations: FMR, ferromagnetic resonance; SPIONs, superparamagnetic iron oxide nanoparticles. 
the ferrofluid of volume $2 \mu \mathrm{L}$ containing a known concentration $(1.4 \mathrm{mM})$.

\section{Results and discussion}

\section{Case I: in vivo study}

\section{Visualization of the SPIONs in the studied tissue}

The results of light microscopy clearly showed the conservation of the morphology of the studied organ in the presence of SPIONs. After 15 minutes of iron nanoparticles administration, Prussian blue-stained structures started to appear within the sinusoid capillaries or between hepatocytes rows. Thereafter, the intensity of Prussian blue-staining increased, indicating a greater number of iron nanoparticle inside macrophages (Figure 3B). Figure 3A shows the micrograph of the liver control sample.

\section{FMR identification of SPIONs}

The iron entities in the liver and blood were investigated at temperatures of 4,100 , and $298 \mathrm{~K}$, firstly focusing on the assignment of the resonances due to iron in the FMR spectra. Each one of the assignments of the FMR lines from liver and blood samples was previously identified by Gamarra and colleagues. ${ }^{25}$ It has been shown that the resonance at $\mathrm{g}=2.1$ is the total contribution of the administered ferrofluid $^{26-28}$ internalized in the cells of the phagocytic mononuclear system such as those of the Kupffer liver cells ${ }^{28}$ and the $\mathrm{Fe}^{+3}$ ions contained in ferritine. ${ }^{29}$ Therefore, the iron resonances in contributions of the FMR spectra from the control samples were subtracted from spectra of the liver and blood samples and thus only the SPIONs contributions were obtained.

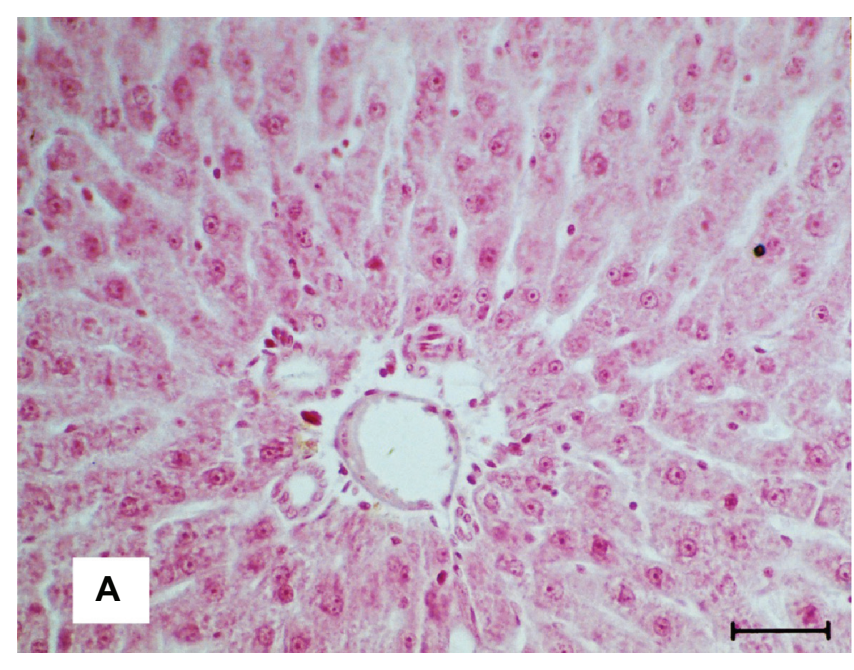

The FMR analysis at the three temperatures showed that the maximum intensity of the broad line at $\mathrm{g}=2.1$ due to the SPIONs present in the liver and blood samples was obtained at $298 \mathrm{~K}$, and therefore this temperature was chosen for the present study.

\section{Analysis of the quantification result by FMR}

The SPIONs concentrations were determined from the areas under the FMR absorption spectra by interpolation in the calibration curve shown in Figure 1. The calculation was performed with the FMR data from samples obtained after the time intervals discussed in the protocol and also from the control sample (see Figures 4 and 6 in Gamarra and colleagues $^{25}$ ). The experimental points represent the average values obtained from FMR measurements of three samples for each condition under investigation.

In a previous study, Gamarra and colleagues observed the close-to-complete disappearance of SPIONs in the blood (see Figure 5 in Gamarra and colleagues ${ }^{25}$ ), starting within $60 \mathrm{~min}$ after the ferrofluid administration. In that work, a first order exponential decay was used for data adjustment. Therefore, nanoparticles concentration $C(t)$ in the blood at a time $\mathrm{t}$ is given by $C(t)=C_{0}^{B} \exp \left(-t / \tau_{B}\right)$, where $C_{0}^{B}$ is the nanoparticles concentration at $t=0$ and $\tau_{B}$ is the characteristic time constant of the nanoparticles elimination from the blood, equivalent to the time required to eliminate $63 \%$ of the administered nanoparticles concentration. The half life time of the nanoparticles elimination from the blood circulation is given by $t_{1 / 2}=\tau_{B}$. In 2 .

In order to corroborate the FMR results for the study of the ferrofluid elimination in the blood, the XRF technique

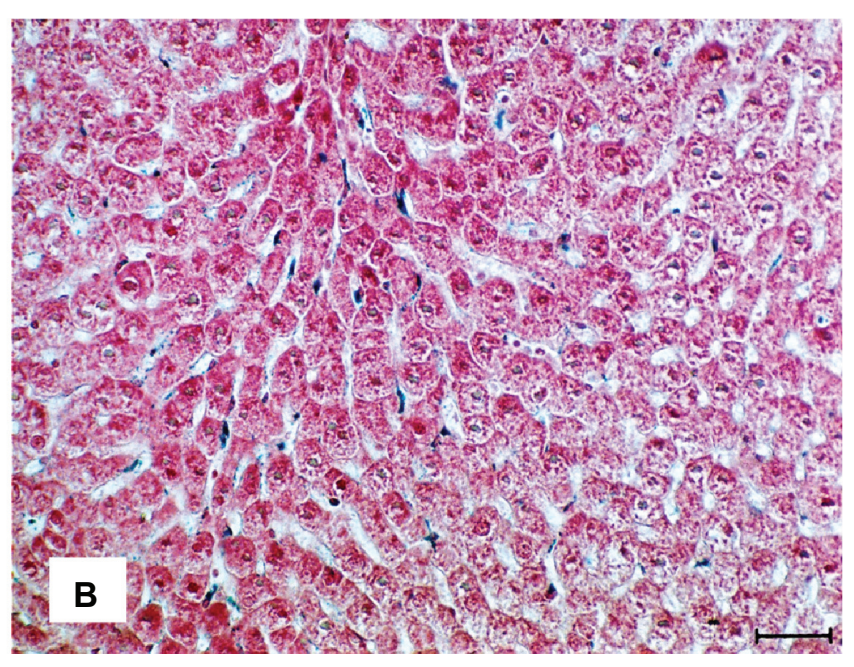

Figure 3 A) Micrograph of liver colored by the Prussian blue from tissues of control animals. B) Micrograph of liver after the SPIONs administration, making evident the presence of the nanoparticles in the tissue; bar $=40 \mu \mathrm{m}$.

Abbreviation: SPIONs, superparamagnetic iron oxide nanoparticles. 


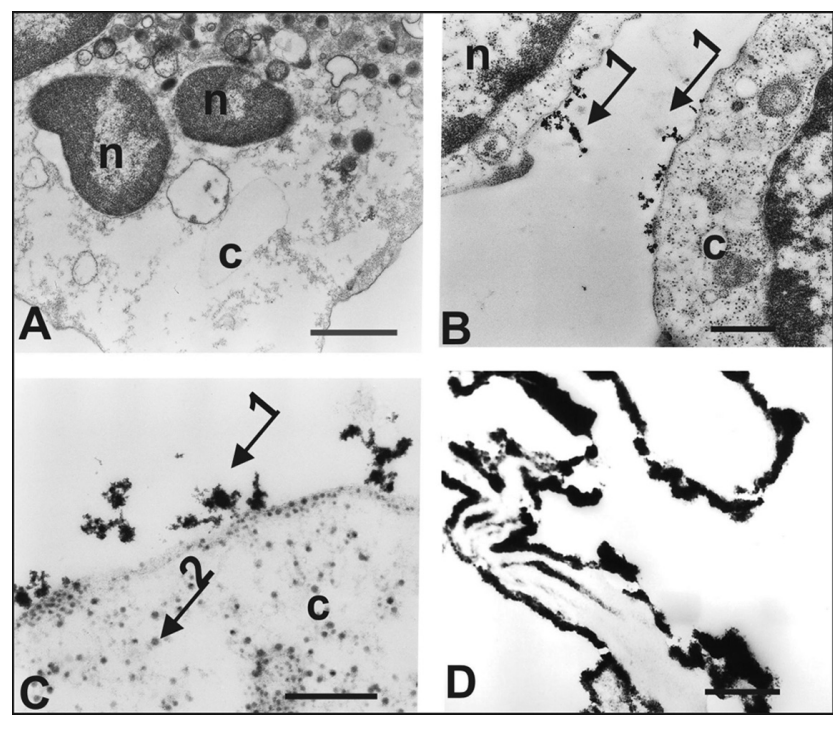

Figure 4 A) TEM of the control stem cells CDI33-. B, C) TEM of the CDI $33^{+}$stem cells labeled with SPIONs. D) TEM of the CDI $33^{+}$cells of not conserved morphology. $\mathrm{n}=$ nucleus, $\mathrm{c}=$ cytoplasm, arrow $\mathrm{I}=$ electrondense granules on the cell surface, arrow 2 = electrondense granules in the cell cytoplasm. Scale: A) $1.0 \mu \mathrm{m}$; B, C) $0.25 \mu \mathrm{m}$;D) $0.5 \mu \mathrm{m}$.

Abbreviations: SPIONs, superparamagnetic iron oxide nanoparticles; TEM, transmission electron microscopy.

was applied to determine the iron concentration in the blood using the calibration curve (Figure 1) of the characteristic iron lines for different periods of time. The values obtained from the curve fitting using both techniques (FMR and XRF) are shown in Table 1.

The study of the biodistribution kinetics of the ferrofluid in the liver using the FMR and XRF techniques was carried out in a similar manner as the study of the sample from the blood. The curves reported by Gamarra and colleagues (see Figure 5 in Gamarra and colleagues ${ }^{25}$ ), obtained from the FMR (continuous line) and XRF (dashed line) suggest that the magnetic nanoparticles are transferred from the blood stream to the liver compartment. It is known that the SPIONs are not only transferred to the liver, but also

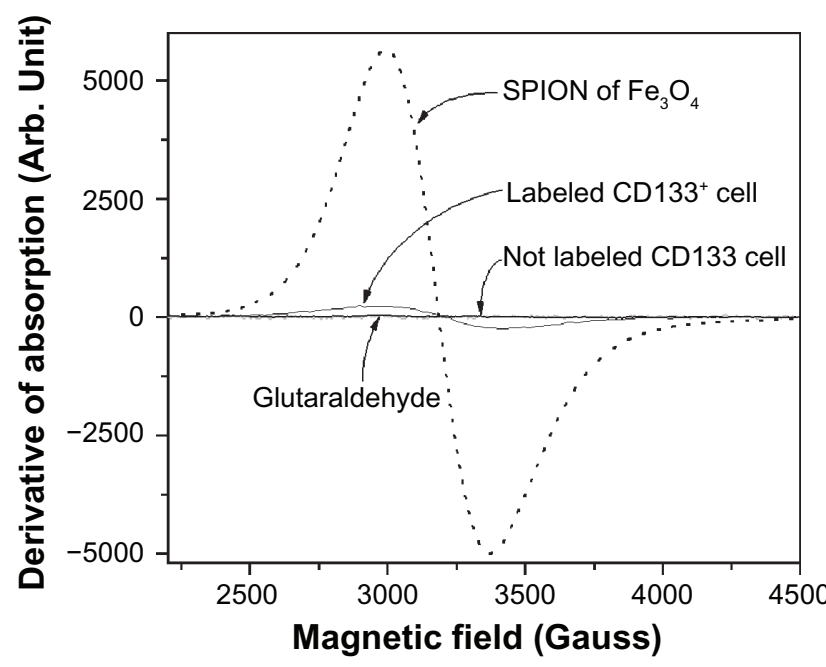

Figure 5 FMR spectra showing the derivative of the absorption of the SPIONs of $\mathrm{Fe}_{3} \mathrm{O}_{4}$, labeled cells, not labeled cells, and the $0.5 \%$ glutaraldehyde fixator.

Abbreviations: FMR, ferromagnetic resonance; SPIONs, superparamagnetic iron oxide nanoparticles.

to other various organs of the rat. ${ }^{30}$ The time dependence of the nanoparticles concentration in the liver is given by $C(t)=C_{0}^{L}\left[1-\exp \left(-t / \tau_{L}\right)\right]$, where $C_{0}^{L}$ is the maximum nanoparticle concentration that the liver would be able to accumulate and $\tau_{L}$ is the characteristic time of the nanoparticles accumulation in the liver.

The FMR and XRF data were adjusted and the equation parameters were calculated and are shown in Table 1. Both studies of the SPIONs elimination from the blood and accumulation in the liver indicate that the results obtained from FMR and those obtained from XRF are consistent.

\section{Case II: in vitro study SPIONs visualization in the labeled cells}

The ultra-structural results obtained by TEM analyses clearly showed the presence of electron-dense granules, indicated by arrow 1, on the surface of CD133+ ${ }^{+}$stem cell (Figures 4B and $\mathrm{C}$ ), therefore revealing the presence of the $\mathrm{AC} 133$

Table I Parameters obtained from the curve adjustments of Figures 5 and 7 of Gamarra and colleagues. ${ }^{25}$ (FMR and XRF data) for the study of the kinetics of elimination from the blood and biodistribution in the liver. Each parameter of the curves was determined from the average value obtained from the results of the measurements of three samples per experimental point

\begin{tabular}{|c|c|c|c|}
\hline & & FMR & XRF \\
\hline \multirow[t]{3}{*}{ Elimination kinetics (blood) } & $C_{0}^{B}\left(\right.$ particles $\left./ \mathrm{mm}^{3}\right)$ & $(7.3 \pm 0.1) \times 10^{12}$ & $(7.8 \pm 0.2) \times 10^{12}$ \\
\hline & $\tau_{B}(\min )$ & $(16.8 \pm 0.8)$ & $(18.2 \pm 0.8)$ \\
\hline & $t_{1 / 2}(\min )$ & $(11.6 \pm 0.6)$ & $(12.6 \pm 0.6)$ \\
\hline \multirow[t]{2}{*}{ Distribution kinetics (liver) } & $C_{0}^{L}\left(\right.$ particles $\left./ \mathrm{mm}^{3}\right)$ & $(32 \pm 1) \times 10^{11}$ & $(31 \pm 1) \times 10^{11}$ \\
\hline & $\tau_{\nu}(\min )$ & $(24 \pm 2)$ & $(19 \pm 2)$ \\
\hline
\end{tabular}

Abbreviations: $F M R$, ferromagnetic resonance; $X R F$, $X$-ray fluorescence. 
monoclonal antibodies coupled to the SPIONs nanoparticles. This finding excludes the control cell (CD133-) which does not exhibit the label on its surface (see Figure 4A). The SPIONs were also observed in the cytoplasm of the stem cell $\mathrm{CD}_{133}{ }^{+}$(Figure 4C), as indicated by arrow 2, suggesting their internalization through the process of endocytosis (pinocytosis).

The previous standardization of this technique has demonstrated a strong electrondense labeling in the periphery of the cellular pellet due to the bad fixation of the material, which explains the reason why the morphology of the cell $\mathrm{CD}_{133}{ }^{+}$was not conserved (Figure 4D).

\section{Analysis of the result of FMR quantification in the cells}

In order to clearly show the expression of the antigenic epitopes (CD133) for the cell of interest, we first obtained only the FMR spectrum of the magnetic beads (Figure 5), ie, the AC133 monoclonal antibody coupled to the magnetic microspheres composed by SPION (Miltenyi Biotec). We observed the resonance at $\mathrm{g}=2.1$ attributed to $\mathrm{Fe}^{3+}$ ions which interact among themselves, showing a superparamagnetic behavior $^{31-33}$ that characterizes the presence of clusters. This signal consists of a strong absorption, whose broad width is attributed to a strong exchange interaction between sufficiently close Fe ions.

The FMR spectrum of the nonlabeled (CD133-) control cells did not present any trace of resonance (Figure 5). Thereafter it was obtained the FMR spectrum of the labeled $\mathrm{CD}_{133}{ }^{+}$cells $\left(0.64 \times 10^{5}\right.$ labeled cells in the volume of $\left.2 \mu \mathrm{L}\right)$ showing a resonance line at $\mathrm{g}=2.1$ (Figure 5) thus indicating the presence of the SPIONs attached to the cells, which were embedded in the $0.5 \%$ glutaraldehyde fixator.

To make sure that the nanoparticles were not present in the fixator, this was removed from the labeled cells by centrifugation (1200 rpm for $10 \mathrm{~min}$ ) and careful aspiration of the supernatant layer. The FMR measurement of the extracted fixator was performed and no signal for the glutaraldehyde was observed, thus indicating the absence of the SPION label in the fixator. This result was confirmed by TEM, being observed that the antibodies coupled to the nanoparticles are attached to the $\mathrm{CD} 133^{+}$cells (Figures 4B, 4C).

To determine the average number of iron particles per cell, it was first determined the number of particles of iron contained in the total number of labeled cells CD133 ${ }^{+}$ $\left(0.64 \times 10^{5}\right)$ in a volume of $2 \mu \mathrm{L}$. The quantification of the iron particles in the total of labeled cells was determined from the area under the absorption curves of the FMR spectra
(Figure 7) and subsequent interpolating in the calibration curve shown in Figure 2, yielding the value of $1.09 \times 10^{-8}$ mole of iron $\left(6.08 \times 10^{5} \mathrm{pg}\right)$ or $4.49 \times 10^{11} \mathrm{Fe}_{3} \mathrm{O}_{4}$ nanoparticles. Consequently, starting from an indirect calculation (taking into account the number of cells contained in $2 \mu \mathrm{L}$ of sample) it was estimated a number of $1.7 \times 10^{-13} \mathrm{~mol}$ of iron $(9.5 \mathrm{pg})$ or $7.01 \times 10^{6}$ iron nanoparticles per cell.

\section{Conclusions}

Superparamagnetic iron oxide nanoparticles have been experiencing a steady increase of their use in biomedical applications, in which cases a detailed characterization of their physicochemical properties, including structural and magnetic properties, is highly desirable. The results presented in this paper are thus part of such an effort. Here we described the methodology for the quantification of SPIONs both in vitro (labeling of stem cells) and in vivo (kinetics of biodistribution in the blood and liver of rats).

One of the fundamental steps in starting the quantitative analysis in both studies is the procedure of qualitatively visualizing the studied samples in order to determine the SPIONs location as well as the conservation of the cells morphology, which will validate the posterior quantification analysis.

Several biological samples contain paramagnetic substances that appear in the FMR spectra, and which do not correspond to the SPIONs. Therefore, it is necessary to identify the resonance lines exclusively due to the SPIONs. In order to assure the correct assignment, it is important to proceed with the subtraction of the resonance spectrum of the sample containing the SPIONs from the spectrum of the control sample.

After that, the SPIONs concentration is determined from the area under the doubly-integrated FMR spectra, whose values are interpolated in an adequate calibration curves. The SPIONs quantification process by FMR for both in vivo and in vitro studies was shown to be a reliable method, enabling us to estimate either the SPIONs content per volume or per cell. These values will be useful for the determination of the administered concentrations of the label and to improve the detection ability of other techniques such as the MRI.

\section{Acknowledgments}

This work was financed by Instituto Israelita de Ensino e Pesquisa Albert Einstein (IIEPAE), and CNPq. AJCF also thanks the Brazilian agency FAPESP. The authors report no conflicts of interest in this work. 


\section{References}

1. McNeil SE. Nanotechnology for the biologist. J Leukoc Biol. 2005;78:585-594.

2. Neuberger T, Schöpf B, Hofmann H, Hofmann M, von Rechenberg B. Superparamagnetic nanoparticles for biomedical applications: Possibilities and limitations of a new drug delivery system. J Magn Magn Mater. 2005;293:483-496.

3. Pankhurst QA, Connolly J, Jones SK, Dobson J. Applications of magnetic nanoparticles in biomedicine. J Phys D Appl Phys. 2003;36: R167-R181.

4. Oberdörster G, Oberdörster E, Oberdörster J. Nanotoxicology: an emerging discipline evolving from studies of ultrafine particles. Environ Health Perspec. 2005;113:823-839.

5. Frank JA, Miller BR, Arbab AS, et al. Clinically applicable labeling of mammalian stem cells by combining superparamagnetic iron oxides and transfection agents. Radiology. 2003;228:480-487.

6. Zhang Z, van de Bos EJ, Wielopolski PA, Jong-Popijus M, Duncker DJ, Krestin GP. High-resolution magnetic resonance imaging of ironlabeled myoblasts using a standard 1.5-T clinical scanner. MAGMA. 2004; 17:201-209.

7. Lacava LM, Lacava ZGM, Da Silva MF, et al. Magnetic resonance of a dextran-coated magnetic fluid intravenously administered in mice. Biophys J. 2001;80:2483-2486.

8. Laçava LM, Laçava ZGM, Azevedo RB, et al. Use of magnetic resonance to study biodistribution of dextran-coated magnetic fluid intravenously administered in mice. J Magn Magn Mater. 2002;252: 367-369.

9. Jain TK, Reddy MK, Morales MA, Leslie-Pelecky DL, Labhasetwar V. Biodistribution, clearance, and biocompatibility of iron oxide magnetic nanoparticles in rats. Mol Pharm. 2008;5:316-327.

10. Bulte JW, Brooks RA, Moskowitz BM, Bryant LHJ, Frank JA. Relaxometry and magnetometry of the MR contrast agent MION-46L. Magn Reson Med. 1999;42:379-384.

11. Bulte JW, Douglas T, Witwer B, et al. Magnetodendrimers allow endosomal magnetic labeling and in vivo tracking of stem cells. Nat Biotechnol. 2002;19:1141-1147.

12. Jendelová $P$, Herynek V, De Croos J, et al. Imaging the fate of implanted bone marrow stromal cells labeled with superparamagnetic nanoparticles. Magn Res Med. 2003;50:767-776.

13. Ali SA, Gene TY, Heather K, et al. Efficient magnetic cell labeling with protamine sulfate complexed to ferumoxides for cellular MRI. Blood. 2004;104:1217-1223.

14. He $\mathrm{G}$, Zhang $\mathrm{H}$, Wei $\mathrm{H}$, et al. In vivo imaging of bone marrow mesenchymal stem cells transplanted into myocardium using magnetic resonance imaging: A novel method to trace the transplanted cells. Int J Cardiol. 2007;114:4-10.

15. Wilhelm C, Gazeau F, Bacri JC. Magnetophoresis and ferromagnetic resonance of magnetically labeled cells. Eur Biophys. 2002;31:118-125.

16. Hinds KA, Hill JM, Shapiro EM, et al. Highly efficient endosomal labeling of progenitor and stem cells with large magnetic particles allows magnetic resonance imaging of single cells. Blood. 2003; 102:867-872.

17. Hill JM, Dick AJ, Raman VK, et al. Serial cardiac magnetic resonance imaging of injected mesenchymal stem cells. Circulation. 2003; 26:1009-1014.
18. Rivière $\mathrm{Ch}$, Boudghène FP, Gazeau F, et al. Iron oxide nanoparticlelabeled rat smooth muscle cells: cardiac MR imaging for cell graft monitoring and quantitation. Radiology. 2005;235:959-967.

19. Weissleder R, Bogdanov A, Neuweltb EA, Papisov M. Long-circulating iron oxides for MR imaging. Adv Drug Deliv Rev. 1995;16:321-334.

20. Jendelová P, Herynek V, Urdzíková L, et al. Magnetic resonance tracking of transplanted bone marrow and embryonic stem cells labeled by iron oxide nanoparticles in rat brain and spinal cord. J Neurosci Res. 2004;76:232-243.

21. Arbab AS, Bashaw LA, Miller BR, et al. Characterization of biophysical and metabolic properties of cells labeled with superparamagnetic iron oxide nanoparticles and transfection agent for cellular MR imaging. Radiology. 2003;229:838-846.

22. Billotey C, Wilhelm C, Devaud M, Bacri JC, Bittoun J, Gazeau F. Cell internalization of anionic maghemite nanoparticles: quantitative effect on magnetic resonance imaging. Magn Reson Med. 2003; 49:646-654

23. Laniado M, Chachuat A. Verträglichkeitsprofil von ENDOREM Radiologe. 1995;35(Suppl. e):S266-S270.

24. Lehner M, Holter W. Endotoxin-free purification of monocytes for dendritic cell generation via discontinuous density gradient centrifugation based on diluted Ficoll-Paque Plus ${ }^{\circledR}$. Int Arch Allergy Immunol. 2002; 128:73-76.

25. Gamarra LF, Pontuschka WM, Amaro JrE, et al. Kinetics of elimination and distribution in blood and liver of biocompatible ferrofluids based on $\mathrm{Fe}_{3} \mathrm{O}_{4}$ nanoparticles: An EPR and XRF study. Mater Sci Eng C. 2008;28:519-525.

26. Lacava LM, Lacava ZGM, da Silva MF, et al. Magnetic resonance of a dextran-coated magnetic fluid intravenously administered in mice. Biophys J. 2001;80:2483-2486.

27. Sharma VK, Waldner F. Superparamagnetic and ferrimagnetic resonance of ultrafine $\mathrm{Fe}_{3} \mathrm{O}_{4}$ particles in ferrofluids. J Appl Phys. 1997;48:4298-4302.

28. Lacava LM, Garcia VAP, Kückelhaus S, et al. Magnetic resonance and light microscopy investigation of a dextran coated magnetic fluid. J Appl Phys. 2003;93:7563-7565.

29. Slawska-Waniewska A, Mosiniewicz-Szablewska E, Nedelko N, Galazka-Friedman J, Friedman A. Magnetic studies of iron-entities in human tissues. J Magn Magn Mater. 2004;272:2417-2419.

30. Weissleder R, Bogdanov A, Neuweltb EA, Papisov M. Longcirculating iron oxides for MR imaging. Adv Drug Deliv Rev. 1995; 16:321-334.

31. Gamarra LF, Brito GES, Pontuschka WM, Amaro JrE, Parma AHC, Goya GF. Biocompatible superparamagnetic iron oxide nanoparticles used for contrast agents: a structural and magnetic study. J Magn Magn Mater. 2005;289:439-441.

32. Hseih CT, Huang WL, Lue JT. The change from paramagnetic resonance to ferromagnetic resonance for iron nanoparticles made by the sol-gel method. J Phys Chem Sol. 2002;63:733-741.

33. Sharma VK, Waldner F. Superparamagnetic and ferrimagnetic resonance of ultrafine $\mathrm{Fe}_{3} \mathrm{O}_{4}$ particles in ferrofluids. J Appl Phys. 1997;48:4298-4302.
International Journal of Nanomedicine

\section{Publish your work in this journal}

The International Journal of Nanomedicine is an international, peerreviewed journal focusing on the application of nanotechnology in diagnostics, therapeutics, and drug delivery systems throughout the biomedical field. This journal is indexed on PubMed Central, MedLine, CAS, SciSearch $\AA$, Current Contents ${ }^{\circledR} /$ Clinical Medicine,

\section{Dovepress}

Journal Citation Reports/Science Edition, EMBase, Scopus and the Elsevier Bibliographic databases. The manuscript management system is completely online and includes a very quick and fair peer-review system, which is all easy to use. Visit http://www.dovepress.com/ testimonials.php to read real quotes from published authors. 